\title{
Approximation of variational problems with a convexity constraint by PDEs of Abreu type
}

\author{
Guillaume Carlier* Teresa Radice ${ }^{\dagger}$
}

April 30, 2018

\begin{abstract}
Motivated by some variational problems subject to a convexity constraint, we consider an approximation using the logarithm of the Hessian determinant as a barrier for the constraint. We show that the minimizer of this penalization can be approached by solving a second boundary value problem for Abreu's equation which is a well-posed nonlinear fourth-order elliptic problem. More interestingly, a similar approximation result holds for the initial constrained variational problem.
\end{abstract}

Keywords: Abreu equation, Monge-Ampère operator, calculus of variations with a convexity constraint.

MS Classification: 35G30, 49K30.

\section{Introduction}

Given $\Omega$, a bounded, open, convex subset of $\mathbb{R}^{d}$ with $d \geq 2, F: \Omega \times \mathbb{R} \rightarrow \mathbb{R}$ strictly convex in its second argument, and $\varphi$ a uniformly convex and smooth function defined in a neighbourhood of $\Omega$, we are interested in the variational problem with a convexity constraint:

$$
\inf _{u \in \bar{S}[\varphi, \Omega]} \mathcal{J}_{0}(u):=\int_{\Omega} F(x, u(x)) \mathrm{d} x
$$

*CEREMADE, UMR CNRS 7534, Université Paris IX Dauphine, Pl. de Lattre de Tassigny, 75775 Paris Cedex 16, FRANCE and INRIA-Paris, MOKAPLAN, carlier@ceremade.dauphine.fr

${ }^{\dagger}$ Department of Mathematics R. Caccioppoli, University of Naples Federico II, Via Cintia-80126, Napoli, ITALY, teresa.radice@unina.it 
where $\bar{S}[\varphi, \Omega]$ consists of all convex functions on $\Omega$ which admit a convex extension by $\varphi$ in a neighbourhood of $\Omega$. This is a way to express in some weak sense the boundary conditions

$$
u=\varphi \text { and } \partial_{\nu} u \leq \partial_{\nu} \varphi \text { on } \partial \Omega
$$

where $\nu$ denotes the outward normal to $\partial \Omega$ and $\partial_{\nu}$ denotes the normal derivative.

Due to the convexity constraint, it is really difficult to write a tractable Euler-Lagrange equation for (1.1) (see [7], [2]). One may therefore wish to construct suitable penalizations for the convexity constraint which force the minimizers to somehow remain in the interior of the constraint and thus to be a critical point of the penalized functional. Since the seminal work of Trudinger and Wang $[9,10]$ on the prescribed affine mean curvature equation, the regularity of convex solutions of fourth-order nonlinear PDEs which are Euler-Lagrange equations of convex functionals involving the Hessian determinant have received a lot of attention. In particular, the Abreu equation which corresponds to the logarithm of the Hessian determinant has been studied by Zhou [11] in dimension 2 and more recently by Chau and Weinkove [3] and Le $[5,6]$ in higher dimensions. What the well-posedness and regularity results of these references in particular suggest is that a penalization involving the logarithm of the Hessian determinant should act as a good barrier for the convexity constraint in problems like (1.1). This was indeed confirmed numerically at a discretized level, see [1].

Our goal is precisely to show that one can indeed approximate (1.1) by a suitable boundary value problem for the Abreu equation. To do so, we first introduce a penalized version of (1.1) with a small parameter $\varepsilon>0$ :

$$
\inf _{v \in \bar{S}[\varphi, \Omega]} \mathcal{J}_{\varepsilon}(v):=\mathcal{J}_{0}(v)-\varepsilon \mathfrak{F}_{\Omega}(v)
$$

where, when $v \in \bar{S}[\varphi, \Omega]$ is smooth and strongly convex, (see section 2 for the definition for an arbitrary $v \in \bar{S}[\varphi, \Omega]), \mathfrak{F}_{\Omega}(v)$ is defined by

$$
\mathfrak{F}_{\Omega}(v):=\int_{\Omega} \log \left(\operatorname{det} D^{2} v\right) .
$$

Using the convexity of $\mathcal{J}_{\varepsilon}$ setting $f(x, u):=\partial_{u} F(x, u)$, one can easily see that if $u$ is smooth and uniformly convex up to $\partial \Omega$, and solves the first-boundary problem for Abreu equation

$$
\varepsilon U^{i j} w_{i j}=f(x, u) \text { in } \Omega, u=\varphi \text { and } \partial_{\nu} u=\partial_{\nu} \varphi \text { on } \partial \Omega
$$


where $w:=\operatorname{det}\left(D^{2} u\right)^{-1}$ and $U$ denotes the cofactor matrix of $D^{2} u$ then it is indeed the solution of (1.3). It turns out however that the second-boundary value problem (where instead of prescribing both values of $u$ and $\nabla u$ one rather prescribes $u$ and $\operatorname{det}\left(D^{2} u\right)$ on $\partial \Omega$ ) is much more well-behaved, see $[3,5,6]$ and it was indeed used as an approximation for the affine Plateau problem in [9]. We shall also consider an extra approximation parameter and a second-boundary value problem on a larger domain and show that it approximates correctly not only (1.3) but also the intial problem (1.1) as the parameter converges to zero.

The paper is organized as follows. Section 2 gives some preliminaries. In section 3 , we show a $\Gamma$-convergence result for $\mathcal{J}_{\varepsilon}$. In section 4 , we consider an approximation by a second boundary value problem on a ball $B$ containing $\bar{\Omega}$, with a further penalization $\frac{1}{\delta}(u-\varphi)$ on $B \backslash \Omega$, for which we prove existence and uniqueness of a smooth solution. In section 5 , we show that when $\delta \rightarrow 0$, we recover the minimizer of the problem from section 3. Finally, we also show full convergence of the second boundary value problem to the initial constrained variational problem (1.1) when $\delta=\delta_{\varepsilon} \rightarrow 0$ as $\varepsilon \rightarrow 0$, provided $F$ satisfies a suitable uniform convexity assumption.

\section{Preliminaries}

In the sequel, $\Omega$ will be an open, bounded and convex subset of $\mathbb{R}^{d}, d \geq 2$. We are also given an open ball $B$ containing $\bar{\Omega}$ and assume that the boundary datum $\varphi$ satisfies for some $\lambda>0$ :

$$
\varphi \in C^{3,1}(\bar{B}), \varphi=0 \text { on } \partial B, D^{2} \varphi \geq \lambda \text { id on } B .
$$

We then define $\bar{S}[\varphi, \Omega]$ as the set of convex functions on $\Omega$, which, once extended by $\varphi$ on $B \backslash \Omega$, are convex on $B$. Note that elements of $\bar{S}[\varphi, \Omega]$ coincide with $\varphi$ on $\partial \Omega$ and are Lipschitz continuous with Lipschitz constant at most $\|\nabla \varphi\|_{L^{\infty}(B)}$ so that $\bar{S}[\varphi, \Omega]$ is compact for the topology of uniform convergence.

Finally, we assume that the integrand $F:(x, u) \in \Omega \times \mathbb{R} \mapsto F(x, u)$ in the definition of $\mathcal{J}_{0}$ in (1.1) is measurable with respect to $x$, strictly convex and differentiable with respect to $u$ and such that that $F(., 0) \in L^{1}(\Omega)$ and $f(x, u):=\partial_{u} F(x, u)$ satisfies $f(., u) \in L^{\infty}(\Omega)$ for every $u \in \mathbb{R}$. These assumptions in particular guarantee that the convex functional $\mathcal{J}_{0}$ is everywhere continuous and Gâteaux differentiable on $\bar{S}[\varphi, \Omega]$.

Following $[9,10,11]$, let us recall how to define $\mathfrak{F}_{\Omega}(v)$ for an arbitrary convex function $v$ on $\Omega$, first recall that the subdifferential of $v$ at $x \in \Omega$ is 
given by

$$
\partial v(x):=\left\{p \in \mathbb{R}^{d}: v(y)-v(x) \geq p \cdot(y-x), \forall y \in \Omega\right\} .
$$

The Monge-Ampère measure of $v$, denoted $\mu[v]$ is then defined by

$$
\mu[v](A):=|\partial v(A)|
$$

for every Borel subset $A$ of $\Omega$. From the seminal results of Alexandrov (see $[4]), \mu[v]$ is indeed a Radon measure and $v \mapsto \mu[v]$ is weakly continuous in the sense that whenever $v_{n}$ are convex functions which locally uniformly converge to $v$ then

$$
\limsup _{n} \mu\left[v_{n}\right](F) \leq \mu[v](F), \forall F \subset \Omega, \text { closed } .
$$

Decomposing the Monge-Ampère measure into its absolutely continuous part and its singular part (with respect to the Lebesgue measure $\mathcal{L}^{d}$ ) as

$$
\mu[v]=\mu_{r}[v]+\mu_{s}[v], \mu_{r}[v] \ll \mathcal{L}^{d}, \mu_{s}[v] \perp \mathcal{L}^{d} .
$$

Thanks to Alexandrov's theorem, $v$ is differentiable twice a.e., at such points of twice differentiability, we denote by $\partial^{2} v$ its Hessian matrix, Trudinger and Wang proved in [9] that $\operatorname{det}\left(\partial^{2} v\right)$ is the density of $\mu_{r}[v]$ with respect to $\mathcal{L}^{d}$, and following their approach, one can define the functional $\mathfrak{F}_{\Omega}$ by

$$
\mathfrak{F}_{\Omega}(v):=\int_{\Omega} \log \left(\operatorname{det} \partial^{2} v(x)\right) \mathrm{d} x, \forall v \in \bar{S}[\varphi, \Omega] .
$$

It is well-known that $\mathfrak{F}_{\Omega}$ is a concave functional and we refer to $[8,9,11]$ for a proof of the useful properties of $\mathfrak{F}_{\Omega}$ recalled below in Lemmas 2.1 and 2.2

Lemma 2.1. The functional $v \in \bar{S}[\varphi, \Omega] \mapsto \mathfrak{F}_{\Omega}(v)$ defined in (2.2) is concave, upper semi-continuous for the topology of local uniform convergence and bounded from above on $\bar{S}[\varphi, \Omega]$ with the explicit bound (where $c_{d}$ denotes the measure of the unit ball of $\mathbb{R}^{d}$ )

$$
\mathfrak{F}_{\Omega}(v) \leq C_{\Omega, \varphi}:=|\Omega| \log \left(\frac{c_{d}\|\nabla \varphi\|_{L^{\infty}}^{d}}{|\Omega|}\right), \forall v \in \bar{S}[\varphi, \Omega] .
$$

As we shall also work on the larger domain $B$, it will be also convenient to consider for every open subset $\omega$ of $B$ and every convex function $u$ on $B$ the concave functional

$$
\mathfrak{F}_{\omega}(v):=\int_{\omega} \log \left(\operatorname{det} \partial^{2} v(x)\right) \mathrm{d} x .
$$

Following the same lines as Lemma 6.4 in Trudinger-Wang [8], we also have: 
Lemma 2.2. If $\omega$ is an open subset of $B$ with $\omega \subset \subset B$, then for every sequence of convex functions $u_{n}$ converging locally uniformly on $B$ to $u$, one has

$$
\limsup _{n} \mathfrak{F}_{\omega}\left(u_{n}\right) \leq \mathfrak{F}_{\omega}(u)
$$

\section{Logarithmic penalization}

Given $\varepsilon>0$, we consider

$$
\inf _{v \in \bar{S}[\varphi, \Omega]} \mathcal{J}_{\varepsilon}(v):=\mathcal{J}_{0}(v)-\varepsilon \mathfrak{F}_{\Omega}(v)
$$

Since $\mathcal{J}_{\varepsilon}$ is strictly convex and lsc on the convex compact set $\bar{S}[\varphi, \Omega]$, we immediately have:

Proposition 3.1. Problem (3.1) admits a unique solution $v_{\varepsilon}$.

Arguing exactly as in $[9,11]$ by using Alexandrov's maximum principle, one can show:

Lemma 3.2. Let $\varepsilon>0$ and $v_{\varepsilon}$ be the solution of (3.1) then $\mu_{s}\left[v_{\varepsilon}\right]=0$ i.e. $\mu\left[v_{\varepsilon}\right]$ has no singular part.

Remark 3.3. Let us remark that Lemma 3.2 enables one to express $-\mathfrak{F}_{\Omega}\left(v_{\varepsilon}\right)$ in an alternative way as the entropy of the push-forward of the Lebesgue measure on $\Omega$ by $\nabla v_{\varepsilon}$. Also, thanks to Lemma 3.2, one can prove uniqueness of the solution of (3.1) when $\mathcal{J}_{0}$ is convex but not necessarily strictly convex.

In dimension 2 , we actually even have a uniform local bound on $\operatorname{det}\left(\partial^{2} v_{\varepsilon}\right)$ :

Proposition 3.4. Let $d=2, \varepsilon>0$ and $v_{\varepsilon}$ be the solution of (3.1), then $\mu\left[v_{\varepsilon}\right]=\operatorname{det}\left(\partial^{2} v_{\varepsilon}\right) \in L_{\mathrm{loc}}^{\infty}(\Omega)$.

Proof. It follows from Theorem 5.1 and Proposition 4.3 that $v_{\varepsilon}$ is the uniform limit as $\delta \rightarrow 0$ of a sequence of smooth functions $\left(v_{\varepsilon}^{\delta}\right)$ in $\bar{S}[\varphi, \Omega]$ such that, for every open subset $\omega$ with $\omega \subset \subset \Omega,\left\|\operatorname{det}\left(D^{2} v_{\varepsilon}^{\delta}\right)\right\|_{L^{\infty}(\omega)} \leq C$ where $C$ is a constant that depends on $\varepsilon$ and $\omega$ but not on $\delta$. By weak convergence of Monge-Ampère measures we deduce that $\operatorname{det}\left(\partial^{2} v_{\varepsilon}\right) \in L_{\mathrm{loc}}^{\infty}(\Omega)$.

Let us now state a $\Gamma$-convergence result for $\mathcal{J}_{\varepsilon}$ : 
Proposition 3.5. The family of functionals $\mathcal{J}_{\varepsilon}$ defined on $\bar{S}[\varphi, \Omega]$ equipped with the topology of uniform convergence $\Gamma$-converges to $\mathcal{J}_{0}$ in particular $v_{\varepsilon}$ converges uniformly to the solution of (1.1).

Proof. Assume $u_{\varepsilon}$ is a family in $\bar{S}[\varphi, \Omega]$ that converges uniformly as $\varepsilon \rightarrow 0$ to $u$, thanks to (2.3) and Fatou's Lemma, we have

$$
\liminf _{\varepsilon} \mathcal{J}_{\varepsilon}\left(u_{\varepsilon}\right) \geq \liminf _{\varepsilon}\left(\mathcal{J}_{0}\left(u_{\varepsilon}\right)-\varepsilon C_{\Omega, \varphi}\right) \geq \mathcal{J}_{0}(u) .
$$

Given $u \in \bar{S}[\varphi, \Omega]$, we now look for a recovery sequence $u_{\varepsilon} \in \bar{S}[\varphi, \Omega]$ converging to $u$ and $\operatorname{such}$ that $\lim \sup _{\varepsilon} \mathcal{J}_{\varepsilon}\left(u_{\varepsilon}\right) \leq \mathcal{J}_{0}(u)$, we simply take

$$
u_{\varepsilon}:=(1-\varepsilon) u+\varepsilon \varphi
$$

since $\partial^{2} u_{\varepsilon} \geq \varepsilon D^{2} \varphi$ we have

$$
\mathfrak{F}_{\Omega}\left(u_{\varepsilon}\right) \geq d|\Omega| \log (\varepsilon)+\int_{\Omega} \log \left(\operatorname{det}\left(D^{2} \varphi\right)\right)
$$

with the convexity of $\mathcal{J}_{0}$, we then have

$$
\left.\limsup _{\varepsilon} \mathcal{J}_{\varepsilon}\left(u_{\varepsilon}\right) \leq \limsup _{\varepsilon}\left((1-\varepsilon) \mathcal{J}_{0}(u)+\varepsilon \mathcal{J}_{0}(\varphi)\right)+O(\varepsilon \log (\varepsilon))\right) \leq \mathcal{J}_{0}(u) .
$$

\section{Second boundary value approximation}

Having Proposition 3.5 in mind, we now fix the value of $\varepsilon$. Throughout this section, to simplify notations, we therefore take $\varepsilon=1$ and we are interested in approximating the solution of

$$
\inf _{v \in \bar{S}[\varphi, \Omega]} \mathcal{J}_{1}(v):=\int_{\Omega} F(x, v(x)) \mathrm{d} x-\mathfrak{F}_{\Omega}(v),
$$

by a second-boundary value problem for Abreu equation. More precisely given $\delta>0$, we consider

$$
U^{i j} w_{i j}=f_{\delta}(x, u), \quad \text { in } B, u=\varphi, w=\psi \text { on } \partial B
$$

where $\psi:=\operatorname{det}\left(\left(D^{2} \varphi\right)^{-1}\right)$ and

$$
f_{\delta}(x, u):=\left\{\begin{array}{l}
f(x, u) \text { if } x \in \Omega \\
\frac{1}{\delta}(u-\varphi(x)) \text { if } x \in B \backslash \Omega
\end{array}\right.
$$


and as before $w=\operatorname{det}\left(D^{2} u\right)^{-1}$ and $U$ is the cofactor matrix of $D^{2} u$. In view of (4.2) and the definition of $f_{\delta}$, it is natural to introduce the functional defined over convex functions on $B$ by

$$
\mathcal{J}_{1}^{\delta}(v):=\int_{\Omega} F(x, v(x)) \mathrm{d} x+\frac{1}{2 \delta} \int_{B \backslash \Omega}(v-\varphi)^{2}-\mathfrak{F}_{B}(v)
$$

where

$$
\mathfrak{F}_{B}(v):=\int_{B} \log \left(\operatorname{det}\left(\partial^{2} v\right)\right)
$$

so that

$$
\mathcal{J}_{1}^{\delta}(v)=\mathcal{J}_{1}(v)+\frac{1}{2 \delta} \int_{B \backslash \Omega}(v-\varphi)^{2}-\int_{B \backslash \Omega} \log \left(\operatorname{det}\left(\partial^{2} v\right)\right) .
$$

\subsection{A priori estimates for the second boundary value problem}

Following a similar convexity argument as in Lemma 2.2 in Chau and Weinkove [3], we first have

Proposition 4.1. Let $u$ be a smooth and uniformly convex solution of (4.2), then

$$
\max _{B}|u|+\int_{\partial B}\left|\partial_{\nu} u\right|^{d}+\frac{1}{\delta} \int_{B \backslash \Omega}|u-\varphi|^{2} \leq C
$$

for some constant $C$ only depending on $B,\|\varphi\|_{C^{3,1}(\bar{B})}$ and the constant $\lambda$ in (2.1).

Proof. First observe that by convexity and (2.1), $u<0$ in $B$ and $\partial_{\nu} u>0$ on $\partial B$. Define $\widetilde{u}:=\varphi, \widetilde{U}$ as the cofactor matrix of $D^{2} \varphi, \widetilde{w}:=\operatorname{det}\left(D^{2} \varphi\right)^{-1}$ and $\widetilde{f}:=\widetilde{U}^{i j} \widetilde{w}_{i j}$ (whose $L^{\infty}$ norm only depends on $\|\varphi\|_{C^{3,1}(\bar{B})}$ and the constant $\lambda$ in (2.1)) we have by the concavity of $\mathfrak{F}_{B},(4.2)$ and the monotonicity of 
$f(x,$.$) :$

$$
\begin{aligned}
0 & \geq\left(\mathfrak{F}_{B}^{\prime}(u)-\widetilde{F}_{B}^{\prime}(\widetilde{u})\right)(u-\widetilde{u}) \\
& =\int_{B}\left(U^{i j} w_{i j}-\widetilde{U}^{i j} \widetilde{w}_{i j}\right)(u-\varphi)+\int_{\partial B} \psi\left(U^{i j}-\widetilde{U}^{i j}\right) \partial_{i}(u-\varphi) \nu_{j} \\
& =\int_{\Omega} f(x, u)(u-\varphi)-\int_{B} \tilde{f}(u-\varphi)+\frac{1}{\delta} \int_{B \backslash \Omega}(u-\varphi)^{2} \\
& +\int_{\partial B} \psi\left(U^{\nu \nu}-\widetilde{U}^{\nu \nu}\right) \partial_{\nu}(u-\varphi) \\
& \geq \int_{\Omega} f(x, \varphi)(u-\varphi)-\int_{B} \tilde{f}(u-\varphi)+\frac{1}{\delta} \int_{B \backslash \Omega}(u-\varphi)^{2} \\
& +\int_{\partial B} \psi\left(U^{\nu \nu}-\widetilde{U}^{\nu \nu}\right) \partial_{\nu}(u-\varphi)
\end{aligned}
$$

where, in the last line, we have used the fact that $\nabla u-\nabla \varphi=\partial_{\nu}(u-\varphi) \nu$ on $\partial B$ and set $U^{\nu \nu}=U \nu \cdot \nu, \widetilde{U}^{\nu \nu}=\widetilde{U} \nu \cdot \nu$. Using the fact that $f(x, \varphi), \widetilde{f}, \varphi$, $\nabla \varphi$ and $\widetilde{U}$ are bounded, we thus get

$$
\frac{1}{\delta} \int_{B \backslash \Omega}(u-\varphi)^{2}+\int_{\partial B} \psi U^{\nu \nu} \partial_{\nu} u \leq C\left(1+\int_{B}|u|+\int_{\partial B} \partial_{\nu} u+\int_{\partial B} U^{\nu \nu}\right) .
$$

Denoting by $R$ the radius of $B$ and by the same argument as in Lemma 2.2 in [3], one has

$$
U^{\nu \nu}=\frac{1}{R^{d-1}} \partial_{\nu} u^{d-1}+E \text { with }|E| \leq C\left(1+\partial_{\nu} u^{d-2}\right) \text { on } \partial B .
$$

Moreover since $u$ is convex and $u=\varphi=0$ on $\partial B$, one has

$$
\max _{B}|u|=-\min _{B} u \leq 2 R \partial_{\nu} u(x) \text { for all } x \in \partial B .
$$

Putting together (4.5), (4.6), (4.7) and the fact that $\inf _{\partial B} \psi>0$, we obtain

$$
\int_{\partial B}\left(\partial_{\nu} u\right)^{d} \leq C\left(1+\int_{\partial B}\left(\partial_{\nu} u\right)^{d-1}\right)
$$

which gives a bound on $\left\|\partial_{\nu} u\right\|_{L^{d}(\partial B)}$ hence also on $\max _{B}|u|$ by (4.7) so that finally the bound on $\delta^{-1} \int_{B \backslash \Omega}(u-\varphi)^{2}$ follows from the latter bounds and (4.5). 


\subsection{Existence and uniqueness of a smooth uniformly convex solution}

Thanks to Theorem 1.1 in [3], a Leray-Schauder degree argument and the a priori estimate (4.4), one easily deduces the following:

Theorem 4.2. For every $\delta>0$, the second boundary value problem (4.2) admits a unique uniformly convex solution which is $W^{4, p}(B)$ for every $p \in$ $[1,+\infty)$.

Proof. Let $D:=\left\{u \in C(\bar{B}),\|u\|_{C(\bar{B})} \leq C+1\right\}$ where $C$ is the constant from (4.4). For $t \in[0,1]$ and $u \in D$, it follows from Theorem 1.1 in [3] that there exists a unique $W^{4, p}$ for every $p \in[1, \infty)$ and uniformly convex solution of

$$
V^{i j} w_{i j}=t f_{\delta}(x, u), w=\operatorname{det}\left(D^{2} v\right)^{-1} \text { in } B, v=\varphi, w=\psi \text { on } \partial B
$$

where $V$ denotes the cofactor matrix of $D^{2} v$. We denote by $v=T_{t}(u)$ the solution of (4.8). Moreover, by Theorem 2.1 of [3], for every $\alpha \in(0,1)$ there are a priori bounds on $\|v\|_{C^{3, \alpha}}$ and on $\sup _{B}\left(\operatorname{det}\left(D^{2} v\right)+\operatorname{det}\left(D^{2} v\right)^{-1}\right)$ that only depend on $C, \alpha, \delta,\|\varphi\|_{C^{3,1}}$ and the constant $\lambda$ in (2.1). Therefore $(t, u) \in[0,1] \times D \mapsto T_{t}(u)$ is continuous on $[0,1] \times D$ and $T_{t}$ is compact in $C(\bar{B})$ for every $t \in[0,1]$. Since $T_{0}$ is constant and by (4.4) it has a unique fixed point in $D$, again by (4.4), $T_{t}$ has no fixed point on $\partial D$, it thus follows from the Leray-Schauder Theorem that $T_{1}$ has a fixed point in $D$, this proves the existence claim for (4.2).

Finally, uniqueness follows from the same argument as in Lemma 7.1 from [10] where it is proven that two smooth solutions actually have the same gradient on $\partial B$ and then are the minimizers of the same strictly convex minimization problem hence coincide.

In dimension $d=2$, following the argument of Remark 4.2 of Trudinger and Wang [9] and taking advantage of the fact that the right-hand side of the Abreu equation (4.2) does not depend on $\delta$ on $\Omega$, we have the following local bound (which we have used in the proof of Proposition 3.4):

Proposition 4.3. Let $d=2$ and $u$ be the solution of (4.2) then for every open set $\omega \subset \subset \Omega,\left\|\operatorname{det}\left(D^{2} u\right)\right\|_{L_{\text {loc }}^{\infty}(\omega)}$ is bounded independently of $\delta$.

Proof. Let $B_{r}:=B_{r}(0) \subset \subset \Omega$, and observe that thanks to (4.4) both $\left\|f_{\delta}(., u(.))\right\|_{L^{\infty}(\Omega)}=\|f(., u(.))\|_{L^{\infty}(\Omega)}$ and $\|\nabla u\|_{L^{\infty}(\Omega)}$ are bounded independently of $\delta$. Define then $\eta(x):=\frac{1}{2}\left(r^{2}-|x|^{2}\right)$ and consider $z:=\log (w)-$ 
$2 \log (\eta)-\frac{1}{2}|\nabla u|^{2}$, by construction $z$ achieves its minimum at an interior point $x_{0}$ of $B_{r}$ at such a point, we have

$$
\frac{\nabla w}{w}=2 \frac{\nabla \eta}{\eta}+D^{2} u \nabla u
$$

We also have

$$
z_{i j}=\frac{w_{i j}}{w}-\frac{w_{i} w_{j}}{w^{2}}+2 \frac{\delta_{i j}}{\eta}+2 \frac{\eta_{i} \eta_{j}}{\eta^{2}}-u_{i j k} u_{k}-u_{i k} u_{j k}
$$

multiplying by $w U=\left[D^{2} u\right]^{-1}$, using $w U^{i j} z_{i j} \geq 0$ at $x_{0}, U^{i j} w_{i j}=f(x, u) \leq C$ and the identities

$$
w U^{i j} u_{i k} u_{j k}=u_{i i}=\Delta u, w U^{i j} u_{i j k} u_{k}=-\frac{w_{k}}{w} u_{k}=-\frac{\nabla w}{w} \cdot \nabla u,
$$

(the second identity is classically obtained by first differentiating the relation $-\log (w)=\log \left(\operatorname{det} D^{2} u\right)$ and then taking the scalar product with $\left.\nabla u\right)$ as well as the fact that $\operatorname{Tr}(U)=\Delta u$ in dimension $d=2$, we get

$$
0 \leq C-w U \frac{\nabla w}{w} \cdot \frac{\nabla w}{w}+2 \frac{w}{\eta} \Delta u+2 w U \frac{\nabla \eta}{\eta} \cdot \frac{\nabla \eta}{\eta}+\frac{\nabla w}{w} \cdot \nabla u-\Delta u .
$$

Using (4.9) and using again $w U=\left[D^{2} u\right]^{-1}$, we then obtain

$$
w U \frac{\nabla w}{w} \cdot \frac{\nabla w}{w}=4 w U \frac{\nabla \eta}{\eta} \cdot \frac{\nabla \eta}{\eta}+D^{2} u \nabla u \cdot \nabla u+4 \frac{\nabla \eta}{\eta} \cdot \nabla u
$$

and

$$
\frac{\nabla w}{w} \cdot \nabla u=2 \frac{\nabla \eta}{\eta} \cdot \nabla u+D^{2} u \nabla u \cdot \nabla u .
$$

Replacing (4.13), (4.14) in (4.12), multiplying by $\eta$ and rearraging gives

$$
\Delta u(\eta-2 w) \leq C \eta-2 \frac{w}{\eta} U \nabla \eta \cdot \nabla \eta-2 \nabla \eta \cdot \nabla u \leq C \eta+\|\nabla \eta\|_{L^{\infty}}\|\nabla u\|_{L^{\infty}(\Omega)} \leq C^{\prime}
$$

If $\eta\left(x_{0}\right) \geq 4 w\left(x_{0}\right),(4.15)$ gives $\eta\left(x_{0}\right) \Delta u\left(x_{0}\right) \leq 2 C^{\prime}$ and since $\Delta u\left(x_{0}\right) w\left(x_{0}\right)^{1 / 2} \geq$ 2 we get the desired lower bound on the minimum of $\eta^{-2} w$. In the remaining case $w\left(x_{0}\right) \geq \frac{1}{4} \eta\left(x_{0}\right) \geq \frac{\eta^{2}\left(x_{0}\right)}{2 r^{2}}$ and we reach the same conclusion. This gives a local lower bound on $w$ i.e. the desired local upper bound on $\operatorname{det}\left(D^{2} u\right)$. 


\section{Convergence}

\subsection{Letting $\delta \rightarrow 0$ for fixed $\varepsilon$}

In this paragraph, we fix $\varepsilon$ (and thus normalize it to $\varepsilon=1$ as we did in the whole of section 4).

Theorem 5.1. Let $u_{\delta}$ be the unique smooth strictly convex solution of (4.2), then $u_{\delta}$ converges uniformly on $\Omega$ to the unique minimizer of (4.1) as $\delta \rightarrow 0^{+}$.

Proof. We already know from (4.4) that (possibly up to an extraction) $u_{\delta}$ converges locally uniformly on $B$ to some convex $u$ and it also follows from (4.4) that $u \in \bar{S}[\varphi, \Omega]$. Let $v \in \bar{S}[\varphi, \Omega]$ (extended by $\varphi$ on $B \backslash \Omega$ ), thanks to (4.2) and the convexity of $\mathcal{J}_{1}^{\delta}$ we first have

$$
\mathcal{J}_{1}^{\delta}(v)-\mathcal{J}_{1}^{\delta}\left(u_{\delta}\right) \geq \int_{\partial B} U_{\delta}^{\nu \nu} \psi \partial_{\nu}\left(u_{\delta}-\varphi\right)
$$

i.e.

$$
\begin{aligned}
\mathcal{J}_{1}(v)-\mathcal{J}_{1}\left(u_{\delta}\right) & \geq \frac{1}{2 \delta} \int_{B \backslash \Omega}\left(u_{\delta}-\varphi\right)^{2}+\int_{B \backslash \Omega}\left(\log \left(\operatorname{det}\left(D^{2} \varphi\right)\right)-\log \left(\operatorname{det}\left(D^{2} u_{\delta}\right)\right)\right) \\
& +\int_{\partial B} U_{\delta}^{\nu \nu} \psi \partial_{\nu}\left(u_{\delta}-\varphi\right) \\
& \geq \int_{B \backslash \Omega}\left(\log \left(\operatorname{det}\left(D^{2} \varphi\right)\right)-\log \left(\operatorname{det}\left(D^{2} u_{\delta}\right)\right)\right)+\int_{\partial B} U_{\delta}^{\nu \nu} \psi \partial_{\nu}\left(u_{\delta}-\varphi\right) .
\end{aligned}
$$

It follows from Lemma 5.2 below that

$$
\liminf _{\delta \rightarrow 0} \int_{B \backslash \Omega}\left(\log \left(\operatorname{det}\left(D^{2} \varphi\right)\right)-\log \left(\operatorname{det}\left(D^{2} u_{\delta}\right)\right)\right) \geq 0 .
$$

We now have to pay attention to the boundary term, we know from (4.6) that $\theta_{\delta}:=\psi U_{\delta}^{\nu \nu}$ satisfies $0 \leq \theta_{\delta} \leq C\left(1+\left(\partial_{\nu} u_{\delta}\right)^{d-1}\right)$ so that thanks to (4.4), $\theta_{\delta}$ is bounded in $L^{\frac{d}{d-1}}(\partial B)$, up to an extraction we may therefore assume that it weakly converges in $L^{\frac{d}{d-1}}(\partial B)$ to some nonnegative function $\theta$. By convexity we also have that for $\tau>0$

$$
\partial_{\nu} u_{\delta}(x) \geq D_{\tau, \nu} u_{\delta}(x):=\frac{1}{\tau}\left(u_{\delta}(x-\tau \nu(x))-u_{\delta}(x-2 \tau \nu(x))\right), \forall x \in \partial B
$$

For small fixed $\tau>0$ note that $D_{\tau, \nu} u_{\delta}$ is bounded independently of $\delta$ thanks to (4.4) and that it converges as $\delta \rightarrow 0$ pointwise to $D_{\tau, \nu} \varphi$, we thus have

$$
\begin{array}{r}
\liminf _{\delta \rightarrow 0} \int_{\partial B} \theta_{\delta} \partial_{\nu}\left(u_{\delta}-\varphi\right) \geq \liminf _{\delta \rightarrow 0} \int_{\partial B} \theta_{\delta}\left(D_{\tau, \nu} u_{\delta}-\partial_{\nu} \varphi\right) \\
=\int_{\partial B} \theta\left(D_{\tau, \nu} \varphi-\partial_{\nu} \varphi\right)
\end{array}
$$


where in the last line we have passed to the limit using the fact that we have the product of a weakly convergent sequence with a strongly convergent sequence. Now letting $\tau \rightarrow 0$ and using the smoothness of $\varphi$, we deduce that

$$
\liminf _{\delta \rightarrow 0} \int_{\partial B} U_{\delta}^{\nu \nu} \psi \partial_{\nu}\left(u_{\delta}-\varphi\right) \geq 0
$$

Since $\mathcal{J}_{1}$ is lower semi-continuous thanks to Lemma 2.2, we can conclude that

$$
\mathcal{J}_{1}(v) \geq \liminf _{\delta \rightarrow 0} \mathcal{J}_{1}\left(u_{\delta}\right) \geq \mathcal{J}_{1}(u)
$$

so that $u$ solves (4.1) and by uniqueness of the minimizer there is in fact convergence of the whole sequence.

In the previous proof we have used:

Lemma 5.2. Let $u_{\delta}$ be the unique smooth strictly convex solution of (4.2) as before, then

$$
\limsup _{\delta \rightarrow 0} \int_{B \backslash \Omega} \log \left(\operatorname{det}\left(D^{2} u_{\delta}\right)\right) \leq \int_{B \backslash \Omega} \log \left(\operatorname{det}\left(D^{2} \varphi\right)\right) .
$$

Proof. The key point here is the estimate $\int_{B} \Delta u_{\delta}=\int_{\partial B} \partial_{\nu} u_{\delta} \leq C$ which follows from (4.4). Let $\omega$ be an arbitrary Borel subset of $B$, we have (for some constant $C$ varying from a line to another):

$$
\begin{aligned}
\int_{\omega} \log \left(\operatorname{det}\left(D^{2} u_{\delta}\right)\right) & \leq C\left(|\omega|+\int_{\omega} \operatorname{det}\left(D^{2} u_{\delta}\right)^{1 / 2 d}\right) \\
& \leq C\left(|\omega|+\int_{\omega} \sqrt{\Delta u_{\delta}}\right) \leq C\left(|\omega|+|\omega|^{1 / 2}\left(\int_{B} \Delta u_{\delta}\right)^{1 / 2}\right) \\
& =C\left(|\omega|+|\omega|^{1 / 2}\left(\int_{\partial B} \partial_{\nu} u_{\delta}\right)^{1 / 2}\right)
\end{aligned}
$$

so that

$$
\int_{\omega} \log \left(\operatorname{det}\left(D^{2} u_{\delta}\right)\right) \leq C\left(|\omega|+|\omega|^{1 / 2}\right) .
$$

Take $0<R^{\prime}<R$ with $\Omega$ contained in $B_{R^{\prime}}$ (recall $R$ is the radius of $B$ ), we then have, thanks to Lemma 2.2 , the fact that $\log \left(\operatorname{det}\left(D^{2} \varphi\right)\right)$ is bounded 
and (5.1):

$$
\begin{aligned}
\limsup _{\delta \rightarrow 0} \mathfrak{F}_{B \backslash \Omega}\left(u_{\delta}\right) & =\limsup _{\delta \rightarrow 0} \mathfrak{F}_{B \backslash \bar{\Omega}}\left(u_{\delta}\right) \\
& \leq \limsup _{\delta \rightarrow 0} \mathfrak{F}_{B_{R^{\prime}} \backslash \bar{\Omega}}\left(u_{\delta}\right)+\underset{\delta \rightarrow 0}{\limsup } \mathfrak{F}_{B \backslash B_{R^{\prime}}}\left(u_{\delta}\right) \\
& \leq \mathfrak{F}_{B_{R^{\prime}} \backslash \bar{\Omega}}(\varphi)+C\left(\left|B \backslash B_{R^{\prime}}\right|+\left|B \backslash B_{R^{\prime}}\right|^{1 / 2}\right) \\
& \leq \mathfrak{F}_{B \backslash \Omega}(\varphi)+C^{\prime}\left(\left|B \backslash B_{R^{\prime}}\right|+\left|B \backslash B_{R^{\prime}}\right|^{1 / 2}\right) .
\end{aligned}
$$

The desired result follows by letting $R^{\prime}$ tend to $R$.

\subsection{Full convergence}

We now take $\delta=\delta_{\varepsilon}>0$ with

$$
\lim _{\varepsilon \rightarrow 0^{+}} \delta_{\varepsilon}=0
$$

i.e. we only have a single small parameter $\varepsilon$ and we consider the secondboundary value problem

$$
\varepsilon U_{\varepsilon}^{i j} w_{i j}^{\varepsilon}=g_{\varepsilon}\left(x, u_{\varepsilon}\right), \quad \text { in } B, u_{\varepsilon}=\varphi, w^{\varepsilon}=\psi \text { on } \partial B
$$

where $\psi:=\operatorname{det}\left(\left(D^{2} \varphi\right)^{-1}\right)$,

$$
g_{\varepsilon}(x, u):=\left\{\begin{array}{l}
f(x, u) \text { if } x \in \Omega \\
\frac{1}{\delta_{\varepsilon}}(u-\varphi(x)) \text { if } x \in B \backslash \Omega
\end{array},\right.
$$

$w^{\varepsilon}=\operatorname{det}\left(D^{2} u_{\varepsilon}\right)^{-1}$ and $U_{\varepsilon}$ is the cofactor matrix of $D^{2} u_{\varepsilon}$. We further assume that there is an $\alpha>0$ such that

$$
(f(x, u)-f(x, v))(u-v) \geq \alpha(u-v)^{2}, \forall(u, v) \in \mathbb{R}^{d}, \text { and a.e. } x \in \Omega
$$

which amounts to say that the integrand $F$ is uniformly convex in its second argument. Under these assumptions, we have a full convergence result:

Theorem 5.3. Let $u_{\varepsilon}$ be the unique smooth strictly convex solution of (5.3), then $u_{\varepsilon}$ converges uniformly on $\Omega$ to the unique minimizer of (1.1) as $\varepsilon \rightarrow 0^{+}$.

Proof. Step 1: a priori estimates. The first step of the proof is similar to the proof of Proposition 4.1. Again define $\widetilde{u}:=\varphi, \widetilde{U}$ as the cofactor matrix 
of $D^{2} \varphi, \widetilde{w}:=\operatorname{det}\left(D^{2} \varphi\right)^{-1}$ and $\widetilde{f}_{\varepsilon}:=\varepsilon \widetilde{U}^{i j} \widetilde{w}_{i j}$. We then have together with (5.4):

$$
\begin{aligned}
0 & \geq \varepsilon\left(\mathfrak{F}_{B}^{\prime}\left(u_{\varepsilon}\right)-\mathfrak{F}_{B}^{\prime}(\widetilde{u})\right)\left(u_{\varepsilon}-\widetilde{u}\right) \\
& \geq \int_{\Omega}\left(f(x, \varphi)-\widetilde{f}_{\varepsilon}\right)\left(u_{\varepsilon}-\varphi\right)+\alpha \int_{\Omega}\left(u_{\varepsilon}-\varphi\right)^{2}+\frac{1}{\delta_{\varepsilon}} \int_{B \backslash \Omega}\left(u_{\varepsilon}-\varphi\right)^{2} \\
& +\varepsilon \int_{\partial B} \psi\left(U_{\varepsilon}^{\nu \nu}-\widetilde{U}^{\nu \nu}\right) \partial_{\nu}\left(u_{\varepsilon}-\varphi\right)
\end{aligned}
$$

thanks to the fact that $f(x, \varphi)-\widetilde{f}_{\varepsilon}$ is bounded uniformly with respect to $\varepsilon$, using Young's inequality and invoking (4.6), we get

$$
\int_{\Omega}\left(u_{\varepsilon}-\varphi\right)^{2}+\frac{1}{\delta_{\varepsilon}} \int_{B \backslash \Omega}\left(u_{\varepsilon}-\varphi\right)^{2}+\varepsilon \int_{\partial B}\left(\partial_{\nu} u_{\varepsilon}\right)^{d} \leq C .
$$

Step 2: convergence. Thanks to (5.5), up to taking a subsequence of vanishing $\varepsilon_{n}$, we may assume that $u_{\varepsilon}$ converges locally uniformly in $B$ to some $u$ such that $u=\varphi$ in $B \backslash \Omega$ so that the restriction of $u$ to $\Omega$ belongs to $\bar{S}[\varphi, \Omega]$. For every $v$ convex on $B$ such that $v=\varphi$ on $\partial B$, define

$$
\widetilde{\mathcal{J}}_{\varepsilon}(v):=\int_{\Omega} F(x, v(x)) \mathrm{d} x+\frac{1}{2 \delta_{\varepsilon}} \int_{B \backslash \Omega}(v-\varphi)^{2}-\varepsilon \int_{B} \log \left(\operatorname{det}\left(\partial^{2} v\right)\right) .
$$

Let then $v \in \bar{S}[\varphi, \Omega]$ (extended by $\varphi$ on $B \backslash \Omega$ ), we then have

$$
\widetilde{\mathcal{J}}_{\varepsilon}(v)-\widetilde{\mathcal{J}}_{\varepsilon}\left(u_{\varepsilon}\right) \geq \varepsilon \int_{\partial B} \psi U_{\varepsilon}^{\nu \nu} \partial_{\nu}\left(u_{\varepsilon}-\varphi\right)
$$

hence

$$
\mathcal{J}_{0}(v) \geq \liminf _{\varepsilon} \mathcal{J}_{0}\left(u_{\varepsilon}\right)+\liminf _{\varepsilon} \varepsilon\left(\mathfrak{F}_{B}(v)-\mathfrak{F}_{B}\left(u_{\varepsilon}\right)\right)-\lim _{\varepsilon} \sup \varepsilon \int_{\partial B} \psi U_{\varepsilon}^{\nu \nu} \partial_{\nu} \varphi
$$

Arguing as in the proof of Proposition 3.5, we may actually assume that $\mathfrak{F}_{\Omega}(v)>-\infty$ so that $\lim \inf _{\varepsilon} \varepsilon \mathfrak{F}_{B}(v) \geq 0$. As for an upper bound for $\varepsilon \mathfrak{F}_{B}\left(u_{\varepsilon}\right)$ we use the fact that thanks to (5.5), we have $\int_{\partial B} \partial_{\nu} u_{\varepsilon} \leq C \varepsilon^{-1 / d}$ and argue in a similar way as in the proof of Lemma 5.2, to obtain

$$
\varepsilon \mathfrak{F}_{B}\left(u_{\varepsilon}\right) \leq C \varepsilon\left(1+\int_{B} \operatorname{det}\left(D^{2} u_{\varepsilon}\right)^{1 / d}\right) \leq C \varepsilon\left(1+\int_{\partial B} \partial_{\nu} u_{\varepsilon}\right) \leq C\left(\varepsilon+\varepsilon^{1-1 / d}\right),
$$

which yields

$$
\liminf _{\varepsilon} \varepsilon\left(\mathfrak{F}_{B}(v)-\mathfrak{F}_{B}\left(u_{\varepsilon}\right)\right) \geq 0
$$


Thanks to (4.6), we have

$$
\int_{\partial B} \psi U_{\varepsilon}^{\nu \nu} \partial_{\nu} \varphi \leq C \int_{\partial B}\left(1+\left(\partial_{\nu} u_{\varepsilon}\right)^{d-1}\right)
$$

but, thanks to (5.5) and Hölder's inequality, we deduce

$$
\varepsilon \int_{\partial B}\left(\partial_{\nu} u_{\varepsilon}\right)^{d-1} \leq C \varepsilon^{\frac{1}{d}}
$$

so that

$$
\mathcal{J}_{0}(v) \geq \liminf _{\varepsilon} \mathcal{J}_{0}\left(u_{\varepsilon}\right)=\mathcal{J}_{0}(u)
$$

hence $u$ solves (1.1) (and the whole family $u_{\varepsilon}$ converges uniformly on $\Omega$ to $u$ by uniqueness of the minimizer of $\mathcal{J}_{0}$ on $\bar{S}[\varphi, \Omega]$ ).

Acknowledgments: G.C. is grateful to the Agence Nationale de la Recherche for its support through the project MAGA (ANR-16-CE40-0014).

\section{References}

[1] Jean-David Benamou, Guillaume Carlier, Quentin Mérigot, and Édouard Oudet. Discretization of functionals involving the MongeAmpère operator. Numer. Math., 134(3):611-636, 2016.

[2] G. Carlier and T. Lachand-Robert. Representation of the polar cone of convex functions and applications. J. Convex Anal., 15(3):535-546, 2008 .

[3] Albert Chau and Ben Weinkove. Monge-Ampère functionals and the second boundary value problem. Math. Res. Lett., 22(4):1005-1022, 2015 .

[4] Cristian E. Gutiérrez. The Monge-Ampère equation, volume 89 of Progress in Nonlinear Differential Equations and their Applications. Birkhäuser/Springer, [Cham], 2016. Second edition [of MR1829162].

[5] Nam Q. Le. Global second derivative estimates for the second boundary value problem of the prescribed affine mean curvature and Abreu's equations. Int. Math. Res. Not. IMRN, (11):2421-2438, 2013.

[6] Nam Q. Le. $W^{4, p}$ solution to the second boundary value problem of the prescribed affine mean curvature and Abreu's equations. J. Differential Equations, 260(5):4285-4300, 2016. 
[7] Pierre-Louis Lions. Identification du cône dual des fonctions convexes et applications. C. R. Acad. Sci. Paris Sér. I Math., 326(12):1385-1390, 1998.

[8] Neil S. Trudinger and Xu-Jia Wang. The Bernstein problem for affine maximal hypersurfaces. Invent. Math., 140(2):399-422, 2000.

[9] Neil S. Trudinger and Xu-Jia Wang. The affine Plateau problem. J. Amer. Math. Soc., 18(2):253-289, 2005.

[10] Neil S. Trudinger and Xu-Jia Wang. Boundary regularity for the MongeAmpère and affine maximal surface equations. Ann. of Math. (2), 167(3):993-1028, 2008.

[11] Bin Zhou. The first boundary value problem for Abreu's equation. Int. Math. Res. Not. IMRN, (7):1439-1484, 2012. 